\title{
First statement on sexual activity in patients with cardiovascular disease
}

The AHA has released a Scientific Statement, endorsed by professional bodies from various clinical specialties, that contains recommendations regarding sexual activity in patients with cardiovascular disease. "This is the first time that this topic has been comprehensively reviewed by a multidisciplinary team on behalf of a major medical organization," remarks Dr Glenn Levine, lead author on the article published in Circulation. "Sexual activity is an important component of quality of life, and integral to many relationships. Many patients with heart disease have fears or concerns about engaging in sexual activity, as do their spouses or partners."

Frequency and satisfaction of sexual activity often decline in patients with coronary artery disease, heart failure, congenital heart disease, or an implanted cardioverter-defibrillator (ICD), and after a myocardial infarction (MI), CABG surgery, or cardiac transplantation because either patients or partners are anxious that sexual activity will worsen the cardiac condition or cause death. Moreover, "both physicians and patients frequently do not discuss issues regarding resumption of sexual activity," says Dr Levine. "The purpose of our Scientific Statement was to systematically review and analyze the data on sexual activity and heart disease, and ... generate recommendations that physicians and patients can utilize to guide counseling and decisions regarding sexual activity."

Overall, the risks of cardiovascular events during sexual activity are low. Coital angina (which occurs minutes or hours after sexual activity) accounts for $<5 \%$ of all angina attacks. Meta-analyses have shown that sexual activity is associated with a 2.7 -fold increased relative risk of MI compared with time not engaged in sexual activity, and that the relative risk of coital MI in sedentary individuals is 3.0-4.4. However, sexual activity is the cause of $<1 \%$ of all acute MIs because exposure to sexual activity is of short duration. In autopsy studies, only

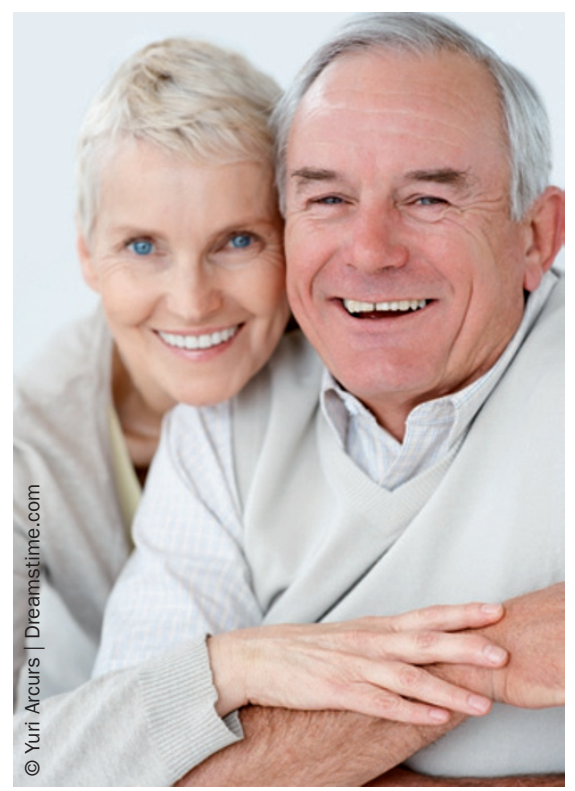

$0.6-1.7 \%$ of sudden deaths were attributed to sexual activity.

The expert consensus was that sexual activity is reasonable in most patients with stable cardiovascular disease, and closely parallels recommendations regarding physical exercise in these patients. Evaluation by thorough patient history, physical examination, and exercise stress testing is reasonable to assess cardiovascular risk. Deferment of sexual activity is recommended in patients with unstable, decompensated, or severe symptomatic cardiovascular disease until their condition is stabilized and optimally managed. Regular exercise and cardiac rehabilitation can reduce the risk of cardiovascular complications with sexual activity.

In patients with coronary artery disease, sexual activity is reasonable for patients with no or mild angina, after uncomplicated MI if no cardiac symptoms present during mild-to-moderate exercise, and after suitable periods of recuperation from complete coronary revascularization by uncomplicated percutaneous coronary intervention or CABG surgery. Patients with incomplete coronary revascularization can be assessed with exercise stress testing, and sexual activity should be deferred in all patients with unstable or refractory angina.

Sexual activity is reasonable in patients with compensated or mild (NYHA class I or II) heart failure, but is not advised in those with decompensated or advanced (NYHA class III or IV) heart failure. In patients with mild or moderate valvular heart disease, a normally functioning prosthetic valve, a successfully repaired valve, or after successful transcatheter valve intervention, sexual activity is reasonable; however, it is not advised in patients with severe or symptomatic valvular disease. Sexual activity is reasonable in patients with a controlled arrhythmia, a pacemaker, or an ICD, as long as moderate physical exercise does not precipitate ventricular tachycardia or fibrillation, or inappropriate ICD shocks. In most patients with congenital heart disease or hypertrophic cardiomyopathy, sexual activity is reasonable unless they are severely symptomatic.

The experts advise that drugs should not be withheld because of their potential impact on sexual function, if they can improve cardiovascular symptoms and survival. Phosphodiesterase-5 inhibitors are useful to treat erectile dysfunction in patients with stable cardiovascular disease, but are contraindicated in patients taking nitrate therapy. Patients with cardiovascular disease should be cautioned about potential adverse effects with unknown ingredients in herbal medications for sexual dysfunction.

Counseling on resumption of sexual activity is recommended for both patients and partners after an acute cardiac event, new diagnosis of cardiovascular disease, or cardioverter-defibrillator implantation.

Gregory B. Lim

Original article Levine, G. N. et al. Sexual activity and cardiovascular disease. A scientific statement from the American Heart Association. Circulation doi:10.1161/CIR.0b013e3182447787 